\title{
Asymmetry Index of Stock Price Fluctuations
}

Weihong Huang* and Yu Zhang

Division of Economics, School of Humanities and Social Sciences, Nanyang Technological University, Singapore

*Corresponding author: Weihong Huang, Economics Division, School of Humanities and Social Sciences, Nanyang Technological University, Singapore, Tel: + 67905733; E-mail: awhhuang@ntu.edu.sg

Rec Date: Jun 27 2014; Acc Date: Jul 24 2014; Pub Date: Jul 312014

Copyright: () 2014 Huang W, et al. This is an open-access article distributed under the terms of the Creative Commons Attribution License, which permits unrestricted use, distribution, and reproduction in any medium, provided the original author and source are credited.

\begin{abstract}
Stock price fluctuation asymmetry, the asymmetry between stock price rise and fall speed, is a general feature of stock indices. Based on inverse statistics, a new measurement, named as asymmetry index, is proposed to evaluate this asymmetry. We calculate and compare asymmetry indices of historical prices from ten stock markets. It is found that in most stock markets, price fall is faster than price rise; while in China and India, price rise is generally faster than price fall.
\end{abstract}

Keywords: Asymmetry index; Inverse statistics; Gain-loss asymmetry; Stock price fluctuation asymmetry

\section{Introduction}

People who have ever invested in stocks may notice that stock price fall is generally faster than stock price rise. This has been mentioned in the Dow Theory, one of the most important origins of technical analyses in financial markets. Hamilton [1] wrote in his famous book, the stock market barometer, and we quote, “... Dow's secondary movement, represented by sharp rallies in a primary bear market and sharp reactions in a primary bull market." Though nowadays the Dow Theory is no longer in fashion, its statement about secondary movements sheds light on our work.

This phenomenon, the asymmetry between stock price rise and fall speed, is referred to as the stock price fluctuation asymmetry in our study. It is a general feature of stock indices. Cont [2] claims that the pattern of large downward movements followed with unmatched smaller upward movements is a stylized fact of stock prices, and names it as the gain-loss asymmetry. Chiang and Stauffer [3] reproduce small upturns and more intermediate downturns by using a Cont-Bouchaud model. Veld camp [4] explains this phenomenon with an endogenous flow of information. Donangelo [5] attribute the gain-loss asymmetry to stocks occasionally synchronizing their short-term draw-downs. Zhang and Li [6] argue that in most cases the gain-loss asymmetry is equivalent to the stock price fluctuation asymmetry and it might be caused by investors' asymmetrically biased risk preferences under positive and negative payoff expectations. Huang and Zheng [7] suggest that fundamentalists' trading behavior contributes to the asymmetry in the probability and the magnitude of price changes.

Though the stock price fluctuation asymmetry has aroused wide interest, most existing studies concentrate on reproducing it and few researchers have proposed any method to assess it quantitatively. There is a reason for this research gap. That is, the timing related to the assessment method could be ambiguous. When studying historical data of stock markets, it is easy to identify the main part of gradual formation and sudden burst of a stock bubble. The tricky part is to decide the exact start time and end time, which can strongly affect the evaluation of the asymmetry. After such a value is calculated, it might be too specific to gain generality as it is based merely on one realization of bubble formation and burst rather than on all available historical data. In our study, we suggest that inverse statistics can provide a proper solution for this problem. And further, an asymmetry index is proposed to evaluate the stock price fluctuation asymmetry.

\section{Inverse Statistics and Asymmetry Index}

Different from usual statistical analysis of financial time series calculating return distribution within fixed time intervals, the method of inverse statistics processes financial time series following an "inverse" procedure. It focuses on the smallest time intervals needed to realize a fixed return. And it produces waiting time distributions for achieving predetermined returns.

Inverse statistics is initially used in Hydrodynamics to describe the spatial distribution of fluid velocity. Ghashghale [8] introduce this method into a study of foreign exchange and make analogies between fully developed turbulence and the price dynamics in foreign exchange markets. Simonsen, Jensen and Johansen have completed a series of studies $[9,10]$ on gain-loss asymmetry and optimal investment horizon in financial markets. Later, inverse statistics has been found as a good classification of mature markets and emerging markets. This draws researchers' attention to the comparison between emerging markets and mature markets. Karpio, Zaluska-Kotur and Orlowski [11] study emerging European markets and proposed a market maturity measurement. Ebadi, Bolgorian and Jafari [12] compare inverse statistics with fractional Brownian motion, and further find that developed markets are more susceptible to exogenous shocks than emerging markets.

Follow the standard procedure of inverse statistics in [6], one can get waiting time distributions and corresponding expected waiting time for achieving predetermined returns. To measure the stock price fluctuation asymmetry, the asymmetry index (hereafter AI) is defined as the difference of price rise and fall speed divided by their sum, as in Equation 1. $\Delta \mathrm{t}$ denotes the waiting time for realizing the predetermined return for the first time. We derive the expected waiting time from waiting time distributions produced by inverse statistics. As the price changes of positive and negative returns share the same absolute value, the AI function can be simplified to Equation 
Page 2 of 2

2. The value of $\mathrm{AI}$ varies from -1 to 1 . A positive $\mathrm{AI}$ indicates that price rise is faster than price fall in general, while a negative AI shows that price fall is faster than price rise. The absolute value of AI implies the intensity of the asymmetry among price rise and fall speed.

Inverse statistics and $\mathrm{AI}$ are related but not exactly the same. The result of inverse statistics is more informative as it contains details of waiting time distributions. AI is a general measurement of stock price fluctuation asymmetry. More importantly, it makes parallel comparison among indices possible.

\section{Results and Discussion}

Table 1 shows data information of ten stock indices and their corresponding AIs. The absolute value of predetermined returns is equal to five times daily volatility in every index. For most indices, their AIs are negative, which means that the price fall is generally faster than price rise in these markets. These negative AIs are consistent with our common perception of gradual formation and sudden burst of price bubbles in stock markets.

\begin{tabular}{|l|l|l|l|l|}
\hline Index & Location & From & To & Al \\
\hline DJIA & US & $10 / 1 / 1928$ & $1 / 26 / 2012$ & -0.0207 \\
\hline FTSE & UK & $4 / 2 / 1984$ & $1 / 26 / 2012$ & -0.1582 \\
\hline FCHI & France & $3 / 1 / 1990$ & $1 / 26 / 2012$ & -0.2151 \\
\hline DAX & Germany & $11 / 26 / 1990$ & $1 / 26 / 2012$ & -0.1327 \\
\hline N225 & Japan & $1 / 4 / 1984$ & $1 / 26 / 2012$ & -0.1589 \\
\hline HSI & Hong Kong & $12 / 31 / 1986$ & $1 / 26 / 2012$ & -0.0459 \\
\hline STI & Singapore & $12 / 28 / 1987$ & $1 / 26 / 2012$ & -0.1766 \\
\hline KS11 & South Korea & $7 / 1 / 1997$ & $1 / 26 / 2012$ & -0.1317 \\
\hline SSEC & China & $1 / 4 / 1993$ & $1 / 20 / 2012$ & 0.0236 \\
\hline BSESN & India & $7 / 1 / 1997$ & $1 / 25 / 2012$ & 0.0307 \\
\hline
\end{tabular}

Table 1: Data analysis of daily close prices of ten indices. Negative asymmetry index (AI) indicates that price fall is generally faster than price rise, and vice versa.

However, the exceptions of SSEC and BSESN are interesting. Positive AIs indicate that price rise is faster than price fall in general. This special feature of stock indices in China and India may reveal great potential of economic growth. Because of the limitation of length, we cannot give a solid support for our conjecture about China and India. Here, we only offer a tentative explanation by searching for other similar examples. We calculated the AI of different historical periods of each index. To avoid the value of AI being affected by fluctuations within business cycles, we made each historical period longer than ten years. We found that the DJIA from 1928 to 1955 has an AI of 0.0622 . We suspected it was not a coincidence that US had blossomed into a strong economic power after the period with a positive AI. We cannot draw a conclusion that the AI can work as an accurate economic probe. But still, we suspect that the special AIs of stock indices in China and India may imply huge potential in these two countries.

As for the robustness, though changing details during data analysis affects the waiting time distributions and further affects the value of $\mathrm{AI}$, our main findings are robust when the predetermined level of return varies from 3 to 20 times daily volatility. The uneven time horizons in data analysis are a compromise we made for more stable AI values.

\section{Conclusion}

In this paper, the stock price fluctuation asymmetry is studied. The inverse statistics is used to analyze the daily close prices of ten most representative indices. The asymmetry index is then developed based on the results of inverse statistics. We find that in most stock markets price fall is faster than price rise. Surprisingly, in China and India, the asymmetry indices show that in general price rise is faster than price fall. We suspect this interesting result might imply huge potential of economic development in China and India.

As a quantitative measurement of stock price fluctuation asymmetry, the asymmetry index makes direct comparison between different stock markets possible. There are other methods to evaluate the results of inverse statistics in existing literature, but none is as simple as the asymmetry index, nor with such a clear economic intuition. In future, we may study the time evolution of asymmetry index, which may reveal more interesting features of stock markets.

\section{References}

1. Hamilton WP (1922) The stock market barometer: a study of its forecast value based on Charles H. Dow's theory of the price movement. Barron's, New York.

2. Cont R (2001) Empirical properties of asset returns: stylized facts and statistical issues. Quantitative Finance 1: 223-236.

3. Chiang I, Stauffer D (2001) Time-reversal asymmetry in Cont-Bouchaud stock market model. Physica A 299: 547-550.

4. Veldkamp LL (2005) Slow boom, sudden crash. Journal of Economic Theory 124: 230-257.

5. Donangelo R, Jensen MH, Simonsen I, Sneppen K (2006) Synchronization model for stock market asymmetry. Journal of Statistical Mechanics 2006: L11001.

6. Zhang Y, Li HG (2011) Investors' risk attitudes and stock price fluctuation asymmetry. Physica A 390: 1655-1661.

7. Huang WH, Zheng HH (2012) Financial crises and regime-dependent dynamics. Journal of Economic Behavior and Organization 82: 445-461.

8. Ghashghale S, Breymann W, Peinke J, Talkner P, Dodge Y (1996) Turbulent cascades in foreign exchange markets. Nature 381: 767-770.

9. Simonsen I, Jensen MH, Johansen A (2002) Optimal investment horizons. European Physical Journal B 27: 583-586.

10. Jensen MH, Johansen A, Petroni F, Simonsen I (2004) Inverse statistics in the foreign exchange market. Physica A 340: 678-684.

11. Karpio K, Zaluska-Kotur MA, Orlowski A (2007) Gain-loss asymmetry for emerging stock markets. Physica A 375: 599-604.

12. Ebadi H, Bolgorian M, Jafari GR (2010) Inverse statistics and information content. Physica A 389: 5439-5446. 